Research Article

\title{
Comparison of Meropenem MIC by E Test and VITEK 2 in resistant Pseudomonas and Acinetobacter isolates
}

\author{
Jananie Kottahachchi, ${ }^{1}$ Joan Faoagali, ${ }^{2}$ Sharon Kleinschmidt ${ }^{2}$ \\ Sri Lanka Journal of Infectious Diseases 2012 Vol.1(2);28-35 \\ DOI: http://dx.doi.org/10.4038/sljid.v2i1.3667
}

Key words: Meropenem; MIC; VITEK 2; Pseudomonas aeruginosa; Acinetobacter baumanii

\begin{abstract}
Pseudomonas aeruginosa and Acinetobacter baumannii cause serious infections in health care institutions. Many isolates are multidrug resistant and sometimes resistant even to meropenem. The minimum inhibitory concentration (MIC) of an antibiotic is useful to decide on specific treatment and several methods of detecting MIC are in current use. Routine application of such methods is cumbersome for clinical laboratories and the newly introduced VITEK 2 automated method is an attractive alternative. The aims of the study were to compare the performance of the E test and the VITEK 2 system in susceptibility testing of resistant strains of P.aeruginosa and A. baumannii to meropenem and to compare the MIC of four different carbapenem antibiotics for P.aeruginosa and A.baumannii. 75 strains of P.aeruginosa and 25 of A.baumanii were selected randomly from the isolate collection of the Princess Alexandra Hospital, Australia. MIC testing using the E test and the VITEK 2 MIC were performed for each isolate according to the manufacturer's instructions and the CLSI guidelines of June, 2010. MICs obtained by VITEK-2 corresponded closely with those obtained with the E test method. Categorical agreement testing for both organisms was $92 \%$ with no major errors and $08 \%$ minor errors. We conclude that VITEK 2 is a reliable method to determine MIC to meropenem for $P$. aeruginosa and $A$. baumanii. Doripenem sensitivity results can be extrapolated from the meropenem sensitivity results.
\end{abstract}

${ }^{1}$ University of Sri Jayewardenepura, Sri Lanka

${ }^{2}$ Princess Alexandra Hospital, Australia

Address for correspondence: Dr. Jananie Kottahachchi. Consultant Microbiologist, Dept of Microbiology, Faculty of Medical Sciences, University of Sri Jayewardenepura, Sri Lanka. Phone:

0772987942 E mail:jananiekottahachchi@yahoo.com 


\section{Introduction}

Pseudomonas aeruginosa and Acinetobacter baumannii have emerged as major human pathogens because of their ability to cause infections in many clinical scenarios. ${ }^{1}$ Infections due to multidrug-resistant P.aeruginosa and A. baumannii are associated with a prolonged hospital stay and increased cost. A. baumannii infections generally affect debilitated patients in intensive care units and are associated with high mortality rates. ${ }^{2}$ Further, A. baumannii is difficult to control and treat because of its prolonged environmental survival and ability to develop resistance to multiple antimicrobial agents. ${ }^{3}$ Inappropriate initial antimicrobial therapy is associated with higher rates of patient morbidity and mortality in infections with P. aeruginosa. ${ }^{4}$ Several studies have documented increasing resistance rates in these two organisms to many antibiotics. ${ }^{5,6}$ As a result, clinicians are left with a restricted choice of therapy, such as the carbapenem antibiotics, including meropenem.

Acquired metallo-beta lactamases (MBL) and Oxa carbapenamases have recently emerged as important resistance mechanisms in P.aeruginosa and A. baumannii limiting therapy with carbapenem antibiotics. ${ }^{7}$ A study by Landman et al $^{8}$ found that about $25 \%$ of P.aeruginosa isolates are resistant to carbapenems and fluroquinolones and over $30 \%$ of A. baumannii are multi drug resistant. The clinical implications of antibiotic resistance are extremely serious and rapid and sensitive diagnostic methods are urgently needed to guide therapy, monitor resistance development and expedite intervention strategies in the management of serious infections, especially among critical care patients.

Genotypic methods are not suitable for routine clinical testing. Therefore determination of antibiotic susceptibility by quantitative MIC (minimal inhibitory concentration) testing is required. Manual methods of antibiotic sensitivity testing are being replaced with automated systems due to the increasing volumes of clinical specimens. The usage of such systems is motivated by the decrease in laboratory turnaround time compared to that required for standardized methods, cost-effectiveness and convenient interfaces with laboratory and hospital information systems which guides the physicians for efficient antimicrobial therapy. The availability of rapid results, reproducibility, ability to trace results, and potential impact on the workflow too favour the use of automatic systems in the microbiology laboratory. However a few studies have demonstrated errors of various automated systems when several organismantimicrobial combinations were tested. ${ }^{9,10}$

VITEK 2 (bioMerieux) is able to determine the MIC and the production of carbapenamase during one test cycle. VITEK 2 detects metabolic changes by fluorescence-based methods and identifies bacteria by monitoring the kinetics of bacterial growth. The MIC phenotype detected for the test isolate is interpolated with all the patterns of the database and the best is identified. VITEK 2 systems have recently been adopted by many microbiological laboratories for rapid identification and the determination of antimicrobial susceptibilities of various types of pathogens, including Gram negative organisms. ${ }^{11}$ The advanced expert system of the instrument detects infrequent or impossible phenotypes of the organisms. 
Given the current increase in infections due to multiresistant P.aeruginosa and A. baumannii infections, it is necessary to confirm the speed and accuracy of the VITEK 2 system in determining MICs for these organisms by comparing its performance with a standard reference method. Epsilometer test (E test) serves as an accurate, easy-to-perform and time-saving alternative to the reference agar and broth dilution methods for quantitative antimicrobial susceptibility testing associated with $P$. aeruginosa.${ }^{12}$ Hence this study was designed to compare the $\mathrm{E}$ test, as the reference method of detecting minimal inhibitory concentration, with the VITEK 2 system for susceptibility testing of resistant $P$. aeruginosa and A. baumannii strains to meropenem and to compare the MICs of four carbapenem antibiotics by the E test method for $P$. aeruginosa and $A$. baumannii.

\section{Methods}

A total of 100 isolates ( 75 P. aeruginosa and 25 A. baumanii complex) were randomly selected from the collection of resistant isolates of the Princess Alexandra Hospital, Queensland, Australia. All organisms had been identified at the time of isolation using the VITEK system. The organisms were revived from storage at $-70^{\circ} \mathrm{C}$ and sub cultured twice on horse blood agar.

VITEK 2 MIC testing was performed for all the isolates according to the manufacturer's instructions. Suspensions of the organism were made in $0.45 \%$ saline and adjusted to the turbidity of 0.5-0.63 McFarland standard using the VITEK 2 Densi Chek densitometer. Suspensions were placed in a VITEK 2 cassette along with a sterile polystyrene test tube and VITEK 2 AST N149 cards containing serial twofold dilutions of 19 antibiotics. The loaded cassettes were placed in the VITEK 2 instrument which automatically processes the isolate until the MICs were obtained. MICs of carbapenem antibiotics other than meropenem could not be evaluated as the VITEK 2 AST N149 card contains only meropenem. Purity of the organism suspensions was ensured by subculture on solid media. E strips (AB bioMerieux, Solna, Sweden) of meropenem, imipenem, ertapenem and doripenem were placed on different Mueller Hinton agar plates inoculated with a $0.5 \mathrm{McF}$ arland standard suspension of the test isolate. Each E-test strip consists of a predefined gradient of antibiotic, allowing for MIC measurements in the range of $0.002-32 \mathrm{ug} / \mathrm{ml}$. According to the manufacturer's instructions, MIC was determined as the value at which the elliptical growth margin intersected the E test strip. P.aeruginosa ATCC 27853 was used as the control for both tests.

MIC's obtained from both methods were interpreted as falling into the susceptible, intermediate or resistant categories as per CLSI document M100-S9, June, 2010. Thus, isolates were considered to be sensitive if the MIC was $\leq 1 \mu \mathrm{g} / \mathrm{ml}$, intermediate if the MIC was $2 \mu \mathrm{g} / \mathrm{ml}$ and resistant if the MIC was $\geq 4 \mu \mathrm{g} / \mathrm{ml}$ for meropenem, imipenem, and doripenem. The breakpoints for ertapenem were $\leq 0.25 \mu \mathrm{g} / \mathrm{ml}, 0.5 \mu \mathrm{g} / \mathrm{ml}$ and $\geq 1 \mu \mathrm{g} / \mathrm{ml}$ respectively.

The MIC results obtained with the VITEK 2 system were compared with those of the reference E test. 


\section{Results}

Table 1 Comparison of MIC obtained using VITEK 2 and E test

\begin{tabular}{|c|c|c|c|c|c|c|c|c|c|c|}
\hline \multirow{3}{*}{$\begin{array}{l}\text { Clinical } \\
\text { category }\end{array}$} & \multirow{2}{*}{\multicolumn{2}{|c|}{$\begin{array}{l}\text { VITEK Z MIC } \\
\text { (meropenem) }\end{array}$}} & \multicolumn{8}{|c|}{ MIC determination by E test } \\
\hline & & & \multicolumn{2}{|c|}{ meгорепет } & \multicolumn{2}{|c|}{ imipenem } & \multicolumn{2}{|c|}{ doripenem } & \multicolumn{2}{|c|}{ ertapenem } \\
\hline & P.аеги & A.bau & Р.деги & A.bau & Р.веги & A.bau & P.аеги & A.bau & Р.авгц & A.bau \\
\hline$S$ & $\begin{array}{c}10 \\
(\mid 3 \%)\end{array}$ & $\begin{array}{c}4 \\
\text { (16\%) }\end{array}$ & $\begin{array}{c}10 \\
(13 \%)\end{array}$ & $\begin{array}{c}2 \\
(8 \%)\end{array}$ & $\begin{array}{c}5 \\
(7 \%)\end{array}$ & $\begin{array}{c}2 \\
(8 \%)\end{array}$ & $\begin{array}{c}19 \\
(25 \%)\end{array}$ & $\begin{array}{c}3 \\
(12 \%)\end{array}$ & $\begin{array}{c}1 \\
(1 \%)\end{array}$ & $\begin{array}{c}2 \\
(8 \%)\end{array}$ \\
\hline I & 0 & $\begin{array}{c}2 \\
(8 \%)\end{array}$ & $\begin{array}{c}6 \\
(8 \%)\end{array}$ & $\begin{array}{c}4 \\
(16 \%)\end{array}$ & $\begin{array}{c}15 \\
(20 \%)\end{array}$ & $\begin{array}{c}4 \\
(16 \%)\end{array}$ & $\begin{array}{c}21 \\
(28 \%)\end{array}$ & $\begin{array}{c}3 \\
(12 \%)\end{array}$ & $\begin{array}{c}1 \\
(1 \%)\end{array}$ & 0 \\
\hline $\bar{R}$ & $\begin{array}{c}65 \\
(87 \%)\end{array}$ & $\begin{array}{c}19 \\
(76 \%)\end{array}$ & $\begin{array}{c}59 \\
(78 \%)\end{array}$ & $\begin{array}{c}19 \\
(76 \%)\end{array}$ & $\begin{array}{c}55 \\
(73 \%)\end{array}$ & $\begin{array}{c}19 \\
(76 \%)\end{array}$ & $\begin{array}{c}35 \\
(47 \%)\end{array}$ & $\begin{array}{c}19 \\
(76 \%)\end{array}$ & $\begin{array}{c}73 \\
(98 \%)\end{array}$ & $\begin{array}{c}23 \\
(92 \%)\end{array}$ \\
\hline
\end{tabular}

$\mathrm{S}$ - sensitive $\mathrm{I}$ - intermediate sensitive $\mathrm{R}$ - resistant

Of the 75 P.aeruginosa isolates, VITEK 2 categorized $10(13 \%)$ as sensitive and $65(87 \%)$ as resistant with no intermediate resistant strains for meropenem. 4 (16\%) of the A. baumannii strains were sensitive, $19(76 \%)$ were resistant and $2(8 \%)$ were of intermediate resistance. Table 1 compares the Vitek 2 MIC clinical categories of organisms to those obtained by the E test.

Discrepancies between VITEK 2 and the E test reference method were classified as very major errors (E-test result was resistant and VITEK 2 result was susceptible), major errors (E-test result was susceptible and VITEK 2 result was resistant), or minor errors (an intermediate susceptibility result was obtained by E test or VITEK 2 method).$^{13}$

VITEK 2 MIC for determination of MIC to meropenem for P.aeruginosa did not show very major errors or major errors in comparison with E test. However $6(8 \%)$ isolates had MIC results that fell into the minor error category. Hence the overall categorical agreement of VITEK 2 meropenem MIC with the reference method was 92\%. A. baumannii VITEK 2 MIC testing with meropenem followed the same pattern with $8 \%$ of minor errors and an overall categorical agreement of $92 \%$ with E test MIC.

\section{Discussion}

Pseudomonas aeruginosa and Acinetobacter baumannii, are multidrug-resistant organisms, increasingly reported as causes of hospital acquired infection worldwide. ${ }^{14}$ These multidrugresistant organisms have diminished susceptibilities to one or more than one of the following groups of antibiotics - antipseudomonal cephalosporins, antipseudomonal carbapenems, $\beta$ lactam- $\beta$-lactamase inhibitor combinations, antipseudomonal fluoroquinolones, and aminoglycosides. ${ }^{15}$ Carbapenems are considered the gold standard of treatment for multidrug resistant $A$. baumannii and $P$. aeruginosa infections. Imipenem has been recognised as the most active agent for treatment of $A$. baumannii infections. ${ }^{2}$ However, recently, strains of these organisms have developed resistance to carbapenems, posing important therapeutic challenges. Peleg and Hooper, ${ }^{16}$ in a recent survey, determined that $26.4 \%$ of P. aeruginosa and $36.8 \%$ of 
A. baumannii causing ventilator-associated pneumonia were resistant to carbapenems (imipenem or meropenem).

Automated antibiotic susceptibility testing systems have been evaluated in numerous studies using several organism-antimicrobial combinations. $P$. aeruginosa has been the organism in which the most performance errors have been reported, especially when tested against the betalactam antimicrobial agents. ${ }^{17,18}$ Hence, we evaluated the ability of the VITEK 2 system to determine the antimicrobial susceptibility of $P$. aeruginosa and A. baumannii isolates accurately using meropenem as the test antibiotic.

A maximum overall category error rate of $10 \%$ should be obtained for a susceptibility test to have acceptable performance, including a maximum of $1.5 \%$ of very major errors and $3.0 \%$ major errors. ${ }^{19}$ In our study, most of VITEK 2 meropenem MIC results were in accordance with reference E test MICs. Both $P$. aeruginosa and A. baumannii recorded $8 \%$ minor error which lies within the acceptable rate of less than $10 \%$ and the overall categorical agreement for MIC for the two types of tested isolates was more than expected at $90 \%{ }^{19}$

Our results are in accordance with the results of a study carried out by Providencia Joyanes et al. $^{20}$ They reported very major errors for $P$. aeruginosa in only one strain $(1.4 \%)$ of $A$. baumannii, for imipenem and not for meropenem. Otto $\mathrm{Krag}^{21}$ tested 224 strains of nonfermentative Gram negatives and their conclusion supports our results that VITEK 2 is suitable for routine clinical use as the overall categorical agreement in antibiotic sensitivity testing was 92.9\%. Minor errors were found in $5.1 \%$ of strains, and major and very major errors were found in $1.6 \%$ and $0.3 \%$ of strains, respectively in the same study. ${ }^{21}$ Annarita Mazzariol et al ${ }^{22}$ demonstrated that VITEK 2 could be used with confidence for identifying resistance to several antimicrobial agents against $P$. aeruginosa including imipenem.

However in contrast to our findings, discordant results were reported in a study using the broth micro dilutions as the reference method, in that VITEK 2 showed very major errors in $19.7 \%$ and minor errors in $34.2 \%$ of $A$. baumanii isolates for amikacin. ${ }^{23}$ Another study demonstrated that the automated systems (MicroScan WalkAway, VITEK 2, and VITEK systems) generally failed to accurately detect piperacillin-tazobactam resistance among clinically significant isolates of $P$. aeruginosa. $^{13}$

In the present study, the MICs of meropenem and imipenem for Pseudomonas aeruginosa and Acinetobacter baumanii were similar, with sensitive, intermediately resistant and resistant rates of $P$. aeruginosa to meropenem being $13 \%, 8 \%$ and $78 \%$ respectively and for imipenem $7 \%$, $20 \%$ and $73 \%$ respectively. For A. baumannii, the rated were $8 \%, 16 \%$ and $76 \%$ respectively for both antibiotics.

Doripenem is a new carbapenem antibiotic with a spectrum of activity comparable to that of imipenem and meropenem. ${ }^{24}$ Considering doripenem MICs in our study, they were more or less similar to the other carbapenems with $P$. aeruginosa strains showing $25 \%$ as sensitive, $28 \%$ as intermediately resistant and $47 \%$ as resistant. $12 \%$ of $A$. baumannii strains were sensitive with $12 \%$ intermediately resistant and $76 \%$ resistant. 
Ertapenem, is a carbapenem that is licenced for once daily use. Although it is reported that $93 \%$ of ESBL-producing coliform isolates are sensitive to ertapenem, ${ }^{25}$ it is not active in infections caused by other resistant organisms, including $A$. baumannii and $P$. aeruginosa. ${ }^{26}$ Theoretically ertapenem is the carbapenem least likely to permeate Gram-negative bacteria rapidly. $98 \%$ of $P$. aeruginosa and $92 \%$ of $A$. baumannii in our study were resistant to ertapenem.

\section{Conclusion}

VITEK 2 meropenem MIC results for P.aeruginosa and A. baumannii correspond closely with those obtained by the reference $\mathrm{E}$ test. Hence it is a reliable method to detect sensitivity to meropenem in the two organisms. Doripenem sensitivity can be extrapolated from that of meropenem. Ertapenem resistance in $P$. aeruginosa and A. baumannii is confirmed.

\section{Acknowledgements}

We would like to thank the Management of the Princess Alexandra Hospital, Queensland, Australia for financial support.

\section{References}

1. Gaynes R, Edwards JR. National Nosocomial Infections Surveillance System. Overview of nosocomial infections caused by Gram-negative bacilli. Clin Infect Dis 2005; 41: 84854. No doi.

2. Tsakris A, Pantazi A, Pournaras S, Maniatis A, Polyzou A, Sofianou D: Pseudo-outbreak of imipenem-resistant Acinetobacter baumannii resulting from false susceptibility testing by a rapid automated system. J Clin Microbiol 2000, 38(9):3505-7. No doi.

3. Poirel L, Nordmann P: Carbapenem resistance in Acinetobacter baumannii: mechanisms and epidemiology. Clin Microbiol Infect 2006, 12(9):826-836. doi:10.1111/j.14690691.2006.01456.x

4. Micek, S. T., A. E. Lloyd, D. J. Ritchie, R. M. Reichley, V. J. Fraser, and M. H. Kollef.. Pseudomonas aeruginosa bloodstream infection: importance of appropriate initial antimicrobial treatment. Antimicrob. Agents Chemother 2005. 49:1306-1311. doi:10.1128/AAC.49.4.1306-1311.2005

5. Streit JM, Jones RN, Sader HS et al. Assessment of pathogen occurrences and resistance profiles among infected patients in the intensive care unit: report from the SENTRY Antimicrobial Surveillance Program. Int J Antimicrob Agents 2004; 24: 111-8. doi:10.1016/j.ijantimicag.2003.12.019.

6. Jones ME, Draghi DC, Thornsberry $\mathrm{C}$ et al. Emerging resistance among bacterial pathogens in the intensive care unit - a European and North American Surveillance study (2000-2002). Ann Clin Microbiol Antimicrob 2004; 3: 14. No doi.

7. Walsh TR, Toleman MA, Poirel L, Nordmann P. Metallo- $\beta$-lactamases, the quiet before the storm? Clin Microbiol Rev 2005;18:306-25. No doi. 
8. David Landman, Simona Bratu, Sandeep Kochar, Monica Panwar, Manoj Trehan, Mehmet Doymaz et al. Evolution of antimicrobial resistance among Pseudomonas aeruginosa, Acinetobacter baumannii and Klebsiella pneumoniae in Brooklyn, NY. J of Antimicrob Chemotherapy; (2007) : 60, 78-82. doi:10.1093/jac/dkm129

9. Doern GV, Brueggemann AB, Perla R, Daly J, Halkias D, Jones RN, et al. Multicenter laboratory evaluation of the bioMérieux Vitek antimicrobial susceptibility testing system with 11 antimicrobial agents versus members of the Family Enterobacteriaceae and Pseudomonas aeruginosa. J Clin Microbiol 1997; 35(8):2115-2119. No doi.

10. Canan Kulah, Elif Aktas, Fusun Comert, Nagihan Ozlu, Isin Akyar and Handan Ankarali Detecting imipenem resistance in Acinetobacter baumannii by automated systems (BD Phoenix, Microscan WalkAway, Vitek 2); high error rates with Microscan WalkAway BMC infectious diseases 2009, 9:30. doi:10.1186/1471-2334-9-30

11. Funke, G., Monnet, D., deBernardis, C., von Graevenitz, A. \& Freney, J. Evaluation of the VITEK 2 system for rapid identification of medically relevant gram-negative rods. $J$ Clin Microbiol 1998; 36: 1948-1952. No doi.

12. Giovanni Di Bonaventura, Evandro Ricci, Nicoletta Della Loggia, Giovanni Catamo, and Raffaele Piccolomini. Evaluation of the E Test for Antimicrobial Susceptibility Testing of Pseudomonas aeruginosa Isolates from Patients with Long-Term Bladder Catheterization. J of Clin Microbiology, March 1998; 36, No. 3: p. 824-826. No doi.

13. Helio S. Sader, Thomas R. Fritsche, and Ronald N. Jones. Accuracy of Three Automated Systems (MicroScan WalkAway, VITEK, and VITEK 2) for Susceptibility Testing of Pseudomonas aeruginosa against Five Broad-Spectrum Beta-Lactam Agents. Journal of Clinical Microbiology, 2006; 44.3:1101-1104. doi:10.1128/JCM.44.3.1101-1104.2006

14. Sunenshine RH, Wright MO, Maragakis LL, Harris AD, Song X, Hebden J, et al. Multidrug-resistant Acinetobacter infection mortality rate and length of hospitalization. Emerg Infect Dis 2007;13: 97-103. No doi.

15. David L. Paterson. The Epidemiological Profile of Infections with Multidrug-Resistant Pseudomonas aeruginosa and Acinetobacter Species. Clin. Infec.Dis. 2006; 43:S43-8. doi:10.1086/504476

16. Anton Y. Peleg and David C. Hooper, Hospital-Acquired Infections Due to GramNegative Bacteria. N Engl J Med 2010; 362:1804-1813. doi:10.1056/NEJMra0904124

17. Burns, J. L., L. Saiman, S. Whittier, J. Krzewinski, Z. Liu, D. Larone et al. Comparison of two commercial systems (Vitek and MicroScan-WalkAway) for antimicrobial susceptibility testing of Pseudomonas aeruginosa isolates from cystic fibrosis patients. Diagn. Microbiol. Infect. Dis. 2001; 39:257-260. No doi.

18. Jones, R. N. Method preferences and test accuracy of antimicrobial susceptibility testing. Arch. Pathol. Lab. Med. 2001;125:1285-1289. No doi.

19. Clinical Laboratory Standard institute, 2008. Development of in vitro susceptibility testing criteria and quality control parameters $8^{\text {th }}$ ed. M23-A3. Clinical Laboratory Standard institute. Wavne. PA. No doi.

20. Providencia Joyanes, Mari’a Del Carmen Conejo, Luis Marti ${ }^{\prime}$ Nez-Marti ${ }^{\prime}$ Nez, and Evelio J. Perea. Evaluation of the VITEK 2 System for the Identification and Susceptibility Testing of Three Species of Nonfermenting Gram-Negative Rods Frequently Isolated from Clinical Samples. J of Clin Microb.2001;39.9:3247-3253. No doi. 
21. Otto-Karg I Validation of Vitek 2 nonfermenting gram-negative cards and Vitek 2 version 4.02 software for identification and antimicrobial susceptibility testing of nonfermenting gram-negative rods from patients with cystic fibrosis. J Clin Microbiol 2009; 47(10): 3283-8. doi:10.1128/JCM.00505-09

22. Annarita Mazzariol, Marco Aldegheri, Marco Ligozzi, Giuliana Lo Cascio, Raffaella Koncan, and Roberta Fontana. Performance of Vitek 2 in Antimicrobial Susceptibility Testing of Pseudomonas aeruginosa Isolates with Different Mechanisms of beta-Lactam Resistance. J of Clin Micro; 2008: 2095-2098. doi:10.1128/JCM.02216-07

23. Dong-Jin Park, Wonkeun Song, Taek-Kyung Kim, Jae-Seok Kim, Han-Sung Kim, Kyu Man Lee. Evaluation of the Vitek 2 AST-N055 Card for the Susceptibility Testing of Acinetobacter baumannii Isolates to Amikacin. Korean J Clin Microbiol. 2009 September; 12(3):144. doi:10.5145/KJCM.2009.12.3.144

24. Elias B. Chahine; Mary J. Ferrill; Mara N. Poulakos Doripenem: A New Carbapenem Antibiotic. American J of Health-System Pharm. 2010;67(23):2015-2024. No doi.

25. Behera B, Mathur P, Das A, Kapil A,. Ertapenem susceptibility of extended spectrum $\beta$ lactamase-producing Enterobacteriaceae at a tertiary care centre in India. Singapore Med J 2009; 50(6) : 628. No doi.

26. Livermore DM, Sefton AM, Scott GM. Properties and potential of ertapenem. $J$ Antimicrob Chemother 2003; 52:331-44. No doi. 